\title{
Hannah Sullivan, The Work of Revision, Cambridge, Harvard University Press, 2013, 360 p.
}

\section{Alienor Vauthey}

\section{(2) OpenEdition}

1 Journals

\section{Édition électronique}

URL : https://journals.openedition.org/genesis/1616

DOI : 10.4000/genesis. 1616

ISSN : 2268-1590

Éditeur :

Presses universitaires de Paris Sorbonne (PUPS), Société internationale de génétique artistique littéraire et scientifique (SIGALES)

Édition imprimée

Date de publication : 9 mai 2017

Pagination : 226-228

ISBN : 979-1023-105636

ISSN : 1167-5101

\section{Référence électronique}

Alienor Vauthey, « Hannah Sullivan, The Work of Revision, Cambridge, Harvard University Press, 2013,

360 p. », Genesis [En ligne], 44 | 2017, mis en ligne le 05 juin 2017, consulté le 18 mars 2023. URL

http://journals.openedition.org/genesis/1616 ; DOI : https://doi.org/10.4000/genesis.1616 
Mais ces feuillets laissent également affleurer des propos plus légers, ancrés dans le temps du tournage et qui pourtant laissent percevoir par exemple l' aplomb de l'écrivain qui amuse ses proches :

Nous allons tourner très peu, vingt plans (rire général), vingt-cinq plans et puis nous savons déjà que le film fera le tour de l'intelligence du cinéma (p. 192).

C'est parce que je ne sais pas jouer que ma musique est tellement belle (p. 192).

Ce volume est donc plus qu'un document sur la vie quotidienne d'un tournage. Il est tout d'abord, un complément à l'édition d'Agatha dans la collection de la «Bibliothèque de la Pléiade», texte édité également par J. Pagès-Pindon sous la direction de Gilles Philippe et paru en 2014. Il constitue une somme passionnante pour le cinéphile qui regarde un film en train de se faire, pour le généticien découvrant le travail de l'écrivain sur des brouillons inédits, pour tout spécialiste ou tout lecteur de Duras qui peut une fois de plus mesurer la proximité de l'oral et de l'écrit chez Marguerite Duras, ou à quel point l'écrit s'inscrit dans l'oral tout en s'affranchissant.

Hannah Sullivan, The Work of Revision, Cambridge, Harvard University Press, 2013, 360 p.

\section{Compte rendu par Alienor Vauthey*}

Dans The Work of Revision, c'est d'abord une histoire que nous raconte Hannah Sullivan : celle de la révision chez les Anglo-Saxons, du XIXe siècle à nos jours. Dans cet intervalle, la révision, d'abord exercée dans la douleur et le secret, deviendrait un gage essentiel de la valeur littéraire du texte, selon une évolution qui prendrait ses racines dans le mouvement moderniste et la culture de l'imprimerie. Par ailleurs, à travers l'analyse de manuscrits, tapuscrits, épreuves et propos métalittéraires de plusieurs grands écrivains modernes anglo-saxons, Sullivan se propose de répondre à la question suivante : «Comment les aspects thématiques et formels d'un texte sont-ils liés à sa genèse ?» (p. 5).

L'auteur de l'étude appuie son travail sur plusieurs écoles de pensée telles que la critique génétique française (on trouve en bibliographie les noms de Pierre-Marc de Biasi, Daniel Ferrer et Louis Hay) et le social-text editing; elle en tire une méthode d'investigation «plus pratique que théorique» (p. 60), qui lui permet de se concentrer sur le geste auctorial sans toutefois négliger les intentions d'autres acteurs ayant pu prendre part à l'élaboration du texte d'une manière ou d'une autre. Le cas le plus marquant est sans aucun doute celui d'Eliot, que les conseils d'Ezra Pound ont amené à supprimer une grande partie de son célèbre poème The Waste Land. Arguant de la grande dispersion des institutions de conservation anglo-saxonnes, Sullivan précise qu'elle a parfois dû se fier à la littérature secondaire plutôt qu'à sa propre analyse des documents. Dans le contexte de ce numéro de Genesis, il importe de souligner que sous le terme de «revision» l'auteur envisage les réécritures aussi bien avant qu' après édition, des ratures du brouillon à celles qu'on peut observer dans les marges d'un livre publié. Plus tard dans l'ouvrage (chap. II), à propos de Dencombe, l'écrivain mis en scène par James dans «The Middle Years», Sullivan propose une passionnante réflexion sur la révision post-éditoriale. Elle oppose alors relecture (rereading), qui altère l'interprétation sans modifier la lettre, et revision, qui destabilise la lettre, parfois dans le but même de refixer le sens. Elle observe alors : «La révision postéditoriale promet à l'auteur la possibilité de se réapproprier le texte alors que celui-ci a déjà commencé à circuler dans la sphère publique et, par conséquent, de refixer sa signification à son point d'origine» (p. 78). Mais elle constate que le désir de réancrer le sens dans l'intention de l'auteur qui motiverait (du moins chez cette figure d'écrivain qu'est Dencombe) la révision chronique conduit, paradoxalement, à une prolifération des versions qui affaiblit l'autorité du texte plutôt qu'elle ne la renforce.
L'histoire de la révision proposée par Sullivan commence avec les romantiques, dont le culte de l'écriture spontanée conduit à condamner par principe un processus qui remet en question l'inspiration première. En ce temps-là, qui plus est, la révision post-éditoriale est une pratique coûteuse. L'invention du linotype en 1886 , qui permet l'accélération de la composition des plaques d'imprimerie et facilite leur réagencement, atténue l'importance de cet obstacle. Le développement de la machine à écrire contribue par ailleurs à stimuler les processus de révision dans les stades prééditoriaux cette fois, puisque, selon Sullivan, «avoir la possibilité de voir les textes fixés sous de nombreuses formes différentes semble promouvoir la fluidité textuelle» (p. 8). À l'aube du XXe siècle, la tendance s'inverse ainsi progressivement : pour les modernistes, grands adeptes de l'expérimentation, les méthodes de révision se présentent comme de bons moyens d'explorer les possibilités d'un texte et de dépasser ce que l'on considère désormais comme la superficialité d'un premier jet. Mais les préjugés ont la peau dure, et la révision demeure encore une pratique seulement à demi avouable. Nombreux sont les auteurs de la première moitié du siècle, comme Woolf, James ou Eliot, qui la pratiquent sans vraiment le confesser. Ce n'est qu' après la guerre que les auteurs se mettent à afficher sans honte leur culte ; tandis que le modernisme institutionnalisé envahit écoles et universités, les processus d'écriture passent sous la loupe; les cours d'écriture créative se mettent à fleurir, qui contribuent à enseigner l'idée qu'il faut réviser. Le fameux slogan de Pound, «make it new », résonne dans les esprits et présente la révision comme un moyen de faire du neuf avec du vieux.

Cette évolution de la pratique de révision soulève une question qui court dans l'ensemble du livre : qu'est-ce que la «bonne» version d'un texte? Pour aborder cette question philologique essentielle,

(*) Université de Lausanne. 
Sullivan compare un stemma traditionnel (plaçant en son sommet une version originale et perdue du texte, idéale parce que non altérée par les erreurs des copistes successifs) et un schéma arborescent dépourvu d'une base unique et dont les branches s'entrecroisent, qu'il conviendrait selon elle d'appliquer à un texte moderne dont les transformations ne sont pas le résultat d'une corruption, mais des multiples opérations de révision effectuées par l'auteur (p. 20). Cette comparaison l'amène à formuler l'hypothèse que si les philologues ont tendance à considérer la première version comme la «bonne», puisque non corrompue, les auteurs modernes de par leur intérêt pour la révision ont au contraire «inversé » la téléologie et considèrent que «le "meilleur" texte est la version unique et finale vers laquelle toutes les autres tendent» (p. 21). Cette dernière affirmation paraît cependant problématique au regard des faits et du schéma donné à voir par l'auteur, qui montre bien qu'il n'existe pas toujours de version unique du texte moderne et que, par suite, son caractère final pose également problème. Sullivan ne manquera pas de s'attaquer à ce paradoxe dans les chapitres II, III et IV.

Successivement consacrés aux trois formes de révision que l'auteur a identifiées en introduction - la substitution, la suppression et l'ajout -, les trois chapitres au cœur de l'ouvrage se concentrent chacun sur l'analyse d'un auteur moderne et d'un texte en particulier : Henry James et «The Middle Years» (1893), T.S. Eliot et The Waste Land (1922), et enfin James Joyce et Ulysses (1918-1920, pour ne mentionner que les dates de premières parutions de chacune de ces trois œuvres). Deux objectifs ici : montrer qu'à chaque méthode de révision correspondent des intentions et des effets distincts - et en passant, ébranler un peu le mythe de la «bonne» version.

L'intérêt d'Henry James pour la substitution dans «The Middle Years », qui change le texte sans en altérer la longueur, préfigurerait l'inclination que montreront plus tard les modernistes pour la suppression et l'ajout, formes de révisions plus téméraires. La substitution microlocalisée ne visant pas à altérer le sens global de l'œuvre mais à en perfectionner le style, Sullivan y lit une tentative de cristalliser une orientation herméneutique unique et toute-puissante du texte à laquelle le lecteur n'aura d'autre choix que de se plier. Privilégiant l'information concentrée et directe, la suppression, à l'exemple du Waste Land d'Eliot, tendrait au contraire à ouvrir un certain nombre de brèches dans lesquelles le lecteur pourra s'engouffrer pour insuffler sa propre interprétation. Quant à l'ajout joycien dans Ulysses, qui comme la suppression peut fonctionner au niveau syntactique comme au niveau narratif, il serait représentatif d'une pratique des auteurs d'après guerre visant à traduire la complexité de la conscience humaine en diluant l'information «utile» dans une masse d'informations digressives et a priori peu pertinentes pour l'intrigue. Il résulte ainsi de la fine analyse des trois textes centraux une typologie pour le moins séduisante, mais dont la valeur de généralité demeure sujette à caution. Au sujet de la «bonne» version du texte, Hannah Sullivan relève le paradoxe jamesien. Dans «The Middle Years », Dencombe relit et révise incessamment son œuvre dans l'espoir vain et délétère d'un aboutissement. La nouvelle qui s'interprète facilement comme une critique du perfectionnisme n'empêchera pas James de procéder exactement de la sorte - sans parvenir plus que son personnage à intéresser les lecteurs à des nouvelles versions de ses œuvres anciennes.

À propos du cas de The Waste Land et de l'intervention de Pound, suggérant à Eliot de supprimer plus d'une centaine de vers, Sullivan regrette que les critiques qui se penchent sur de tels faits tendent à les considérer systématiquement comme un heureux nettoyage. Les déclarations d'Eliot vont dans le même sens. Sullivan n'est pas de cet avis et pense que les révisions de Pound ont contribué à conférer au poème l'apparence déstructurée dont se plaignait Eliot dans les années qui ont suivi. $\mathrm{Au}$ fond, pour Sullivan, Pound n'a fait que donner au poème l'une de ses multiples formes possibles.

L'avant-dernier chapitre aborde la question de la révision sous l'angle du genre en s'intéressant aux récits partiellement ou entièrement autobiographiques et à l'écriture rétrospective (Virginia Woolf, To the Lighthouse, «A Sketch of the Past », Leslie Stephen, The Mausoleum Book et James Joyce, A Portrait of the Artist as a Young Man). Partant du principe que l'autobiographie est le genre le plus susceptible d'être soumis à révision - car cesser de réviser équivaudrait à dire «je suis mort»-, Hannah Sullivan ne cherche non plus seulement à expliquer les effets de telle ou telle forme de révision sur le texte et son sens, mais à comprendre pourquoi les auteurs révisent. Pourquoi Virginia Woolf reviendra-t-elle sur son enfance dans «A Sketch» après y avoir mis un point final dans To the Lighthouse? Ce n'est plus le geste de l'auteur qui dévoile quelque chose du texte, mais le parcours génétique du texte qui révèle quelque chose de l'auteur. De fait, la révision dans le genre autobiographique a tendance à intervenir de manière plus massive sur les épisodes traumatiques et s'apparenterait donc à un processus de cure qui permettrait à l'auteur de revoir un souvenir et d'en combattre le trauma.

Hannah Sullivan clôt son ouvrage en «se risquant à» (p. 257) l'hypothèse que la culture digitale du XXIe siècle, qui a vu se multiplier les technologies permettant de réviser très simplement et très fréquemment, va non pas rendre la révision plus probable, mais au contraire la rendre moins nécessaire puisque par définition plus aucun texte ne sera jamais réellement fixé à un moment ou à un autre. Elle fait également écho dans ce dernier chapitre à des préoccupations déjà soulevées de nombreuses fois par la critique génétique, faisant remarquer par exemple que le fichier Word qui conserve la trace des changements effectués sur un texte est particulièrement ennuyeux à lire, sans parler du fait qu'il retient même les changements effectués par une autre personne que l'auteur sans possibilité de distinction. 
Comment s'adapter à ce nouveau type de matériel? La définition même de ce qu'est un texte - et avec elle, la critique génétique - ne va-t-elle pas devoir évoluer? Pour Sullivan, la pratique de la révision a atteint son point d'orgue avec la culture de l'imprimerie, et réviser «passionnément» et «indéfiniment» aujourd'hui n'est au fond plus qu'un «exercise in nostalgia» (p. 269).
En abordant la révision dans la littérature anglo-saxonne sur deux siècles, The Work of Revision ne manquera pas de surprendre les généticiens français, habitués à traiter de corpus beaucoup plus restreints, de plus près et toujours à partir des sources primaires. Les simplifications requises par un exercice de ce genre font à la fois sa force et sa faiblesse. Les hypothèses typologiques formulées ici, ainsi que la proposition de périodisation, méritent d'être éprouvées par d'autres études de cas, ou comparées à l'évolution des pratiques dans d'autres traditions littéraires. L'ouvrage aurait alors atteint l'objectif qu'il se donne : contribuer au champ de la «critique génétique comparative» (p. 59). 